\title{
A PERCEPÇÃO DE VIDA DOS OSTOMIZADOS NO ÂMBITO SOCIAL*
}

\author{
Jordania Calixto da SILVA ${ }^{1}$ \\ Maria Cidney SOARES ${ }^{2}$ \\ Halley de Sousa ALVES ${ }^{3}$ \\ Giulianna Soares GARCIA ${ }^{4}$
}

\begin{abstract}
Recebido em: 15/05/2014 - Aprovado em: 30/06/2014 - Disponibilizado em: 30/07/2014
\end{abstract}
\begin{abstract}
RESUMO: As ostomias, ostomas, estomas ou estomias são derivados do grego e significam "boca" ou "abertura". Correspondem à exteriorização de vísceras ocas através do corpo, e são realizados, cirugicamente, devido a doenças congênitas, tumores, câncer intestinal, traumatismo e doenças inflamatórias. Esta condição, associada ao uso das bolsas coletoras, pode causar constrangimento, vergonha e dificuldade no convívio social ao indivíduo que a possui. Neste sentido, esta pesquisa teve como objetivo investigar as mudanças ocorridas na vida após a colostomia. Trata-se de um estudo exploratório e descritivo, com abordagem qualitativa, realizado na Associação dos Ostomizados do Agreste e Sertão da Paraíba no mês de agosto de 2009, onde foram entrevistados todos os associados, tendo em vista a questão norteadora "mudanças ocorridas na vida". Os depoimentos foram submetidos à análise temática do conteúdo, onde emergiram três categorias: "Discriminação", "Vida sexual" e "Alimentação". A análise demonstrou que os ostomizados enfrentam, no seu cotidiano, muita dificuldade de adaptação no âmbito social.

DESCRITORES: Ostomia; Colostomia; Adaptação; Enfermagem; Discriminação.
\end{abstract}

\section{THE PERCEPTION OF LIFE OF OSTOMATES IN THE SOCIAL SPHERE}

ABSTRACT: Ostomies, ostomas, stomas or stomata are derived from Greek and means "mouth" or "opening". They correspond to the manifestation of hollow viscera through the body, and are performed surgically due to congenital diseases, tumors, intestinal cancer, trauma and inflammatory diseases. This condition, associated with the use of collection bags, can cause embarrassment, shame and difficulty in socializing the individual who possesses it. In this sense, this research aimed to investigate the changes in life after colostomy. This is an exploratory and descriptive study with a qualitative approach, performed in Ostomy Association of the Wasteland and Hinterland of Paraíba in August 2009 to interview all members, with a view to guiding question "changes in life". The reports were submitted to thematic content analysis which revealed three categories: "Discrimination", "sex life" and "Power". The analysis showed that ostomates face in their daily lives, much difficulty in adapting the social sphere.

DESCRIPTORS: Ostomy; Colostomy; Adaptation; Nursing; Discrination.

\section{LA PERCEPCIÓN DE LA VIDA DE LOS OSTOMIZADOS EN EL ÁMBITO SOCIAL}

RESUMEN: Ostomías, ostomas, estomas o estomas se derivan del griego y significa "boca" o "apertura". Se corresponden con la manifestación de la víscera hueca a través del cuerpo, y se llevan a cabo quirúrgicamente debido a enfermedades congénitas, tumores, cáncer intestinal, traumatismos y enfermedades inflamatorias. Esta condición, asociada con el uso de bolsas de recogida, puede causar vergüenza, la vergüenza y la dificultad en la

\footnotetext{
* Artigo desenvolvido a partir de Monografia de Graduação em Enfermagem intitulada: Ostomizados e o autocuidado: percepção dos usuários de uma associação para colostomizados no município de Campina Grande - PB, apresentada à Faculdade de Ciências Médicas - FCM, em 2009.

${ }^{1}$ Enfermeira. Especialista em Saúde da Família pela Faculdade Montenegro. Especialista em Auditoria em Saúde pela FIP - Faculdades Integradas de Patos. E-mail: jordaniacalixto@msn.com;

${ }^{2}$ Enfermeira. Mestre em Enfermagem. Professora da Faculdade de Ciências Médicas de Campina Grande. Membro do Grupo de Estudo e Pesquisa em Saúde, Mulher e Gênero - GEPSAM/UFPB. E-mail: profcidneysoares@ hotmail.com;

${ }^{3}$ Bacharel em Odontologia pela Universidade Estadual da Paraíba - UEPB. E-mail: halleyss@ hotmail.com;

${ }^{4}$ Enfermeira. Mestranda em Políticas Públicas pela Universidade Federal de Pernambuco (UFPE). E-mail: garcia.giulianna@gmail.com.
} 
socialización de la persona que lo posee. En este sentido, esta investigación tuvo como objetivo investigar los cambios en la vida después de la colostomía. Se trata de un estudio exploratorio y descriptivo, con abordaje cualitativo, realizado en la Asociación de Ostomía del Yermo y Hinterland de Paraíba en agosto de 2009 para entrevistar a todos los miembros, con el fin de orientar los cambios de interrogación "en la vida". Los informes fueron sometidos a análisis de contenido temático que reveló tres categorías: "Discriminación", "vida sexual" y "Power". El análisis mostró que los ostomizados se enfrentan en su vida cotidiana, muchas dificultades en la adaptación de la esfera social.

DESCRIPTORES: Ostomía; La colostomía; Adaptación; Enfermería; Discriminación.

\section{INTRODUÇÃO}

As ostomias, ostomas, estomas ou estomias são derivados do grego e significam "boca" ou "abertura". Correspondem à exteriorização de vísceras ocas através do corpo, devido a doenças congênitas, tumores, câncer intestinal, traumatismo e doenças inflamatórias (GEMELLI; ZAGO, 2002). Dependendo da parte do corpo onde será feito o procedimento, adquirem um termo específico (SANTOS, 2005).

Os estomas intestinais, que podem ser indicados em alguma porção intestinal, recebem os seguintes termos: colostomias (procedimento realizado no colón ascendente, transverso, descendente ou sigmóide); ileostomias (procedimento realizado no íleo); e cecostomia (procedimento realizado no ceco)(SANTOS, 2005).

Os estomas se apresentam normalmente com cor róseoavermelhado, úmido e sem quaisquer sensações ao toque; além disso, sangram discretamente quando sofrem atrito e apresentam eliminação fecal involuntária (NETTINA, 2003).
As ostomias intestinais têm por finalidade desviar o conteúdo fecal através de um estoma, confeccionado cirurgicamente no músculo reto abdominal. Pode ser definitivo ou temporário, continente (quando o paciente tem controle de suas evacuações) ou incontinente (quando o paciente não tem controle de suas evacuações), dependendo da causa que levou ao determinado procedimento (CESARETTI et al., 2005).

Esses conteúdos intestinais eliminados para fora do corpo são armazenados em um dispositivo ou bolsa de drenagem, que devem ser trocados rotineiramente (CESARETTI et al., 2005).

A consistência das fezes depende da localização das ostomias. Nas ileostomias, devido ao seu desvio total do intestino grosso, as fezes se apresentam mais líquidas e mais frequentes. O mesmo ocorre com as colostomias do cólon ascendente. As colostomias do cólon transverso apresentam fezes mais sólidas e formadas. Já a colostomia do cólon 
sigmóide elimina fezes normais

(POTTER; PERRY, 2004).

Segundo Swaringen e Howard (2005), os tipos de ostomias mais comumente realizadas são: colostomias do cólon descendente ou sigmóide e ileostomias.

As colostomias do cólon descendente ou sigmóide são realizadas preferencialmente em pacientes com idade igual ou superior a 40 anos. O estoma fica localizado no quadrante superior esquerdo e poderá se apresentar plano ou protuso. São indicadas em casos de câncer do sigmóide ou reto, diverticulite crônica, anomalias congênitas ou traumas (SWARINGEN; HOWARD, 2005).

Já a ileostomia é indicada em casos de doença de Cronh, câncer, traumas e polipose familiar. Essa cirurgia é realizada com maior freqüência em pacientes adolescentes. $\mathrm{O}$ estoma fica localizado no quadrante inferior esquerdo e apresenta protusão. Este procedimento cirúrgico também adota o sistema de bolsa coletora (SWARINGEN; HOWARD, 2005).

A avaliação pré-operatória, realizada por um estomoterapeuta, é indispensável para a família e para o cliente submetido ao ato cirúrgico, pois, por meio dela, investiga-se os níveis de conhecimento do paciente e da sua família acerca do diagnóstico e tratamento (CESARETTI et al., 2005).

Em muitas ocasiões, o indivíduo encontra-se nervoso ou ansioso diante do procedimento a ser realizado, e isso pode atingir a família (MENDONÇA et al., 2007). O enfermeiro deve intervir, pois o repasse de informações é imprescindível para a diminuição do grau de preocupação (SCHIMIDT; ARRUDA, 2012).

Outro ponto que merece destaque é a marcação do estoma, pois uma área que permita aderência do dispositivo e visualização do paciente pode prevenir complicações. É importante que se verifique o "tipo de ostoma, marcar claramente o local, localizar o músculo reto abdominal, quando em dúvida marcar duas localizações, atentar para atividade de trabalho, lazer e práticas esportivas" (MENDONÇA et al., 2007).

Com relação à avaliação e estabilidade da dieta dos ostomizados, é importante ressaltar que estas devem ser feitas por nutricionistas no período pré, intra e pós-operatório, pois são de grande importância para a regulação, controle de gases e eliminação fecal (DUGAS, 1998).

De acordo com Dias e Teixeira (2005), a dieta deve ser restrita em resíduos, para que se evite a diarréia, e rica em fibras, para se ter uma regulação intestinal. Além disso, o consumo de 
alimentos como cebola e repolho devem ser evitados, pois aumentam a produção de gases.

Esses autores ainda destacam que é de suma importância a inserção dos ostomizados nos grupos de autoajuda. $\mathrm{O}$ convívio com outras pessoas ostomizadas minimiza o sofrimento, pois há a troca de conhecimentos acerca da ostomia.

Por fim, é oportuno destacar o autocuidado. De acordo com a teoria do autocuidado e do déficit do autocuidado de Orem, os indivíduos devem participar de um processo educacional para que se tornem aptos a se cuidar. Quando isso não ocorre, por qualquer que seja o motivo, há necessidade da atuação de enfermagem (MENDONÇA et al., 2007).

Tendo em vista toda a complexidade que envolve a confecção de uma ostomia, o objetivo deste estudo é investigar a percepção e as mudanças ocorridas na vida após a colostomia dos pacientes ostomizados cadastrados numa Associação dos Ostomizados do Agreste e Sertão da Paraíba.

\section{MEDOTOLOGIA}

Trata-se de um estudo
exploratório e descritivo, com
abordagem qualitativa.

As pesquisas exploratórias têm como objetivo o levantamento de dados, as entrevistas com os participantes e a análise dos dados obtidos. Sua principal característica é a descrição da população quanto à idade, sexo, nível de escolaridade, entre outros, utilizando técnicas padronizadas como o questionário e a observação sistemática (GIL, 2002).

A abordagem qualitativa foi escolhida por trabalhar com um universo de significados, motivos, aspirações, crenças, valores e atitudes, correspondendo à profundidade das relações sociais (MINAYO, 2006).

A pesquisa foi realizada na ASSOASP (Associação dos Ostomizados do Agreste e Sertão da Paraíba), localizada no município de Campina Grande- PB, tendo como público alvo os pacientes ostomizados cadastrados na associação. Os participantes da pesquisa foram identificados, ao longo do artigo, com letras do alfabeto, para garantia do anonimato quanto à identidade.

Os dados foram coletados durante o mês de agosto de 2009. Foram entrevistados 20 pacientes cadastrados na ASSOASP, tendo como critérios de inclusão: ser portador de colostomia; ser maior de 18 anos; realizar tratamento/acompanhamento no período do estudo; e concordância em participar da pesquisa. Todos os participantes 
assinaram o Termo de Consentimento

Livre e Esclarecido.

Para a coleta de dados utilizou-se a entrevista semiestruturada, pois esta permite caracterizar a subjetividade e a particularidade dos discursos. Os pacientes foram analisados de forma individual através da técnica de análise de conteúdo do tipo temática, que busca o mesmo sentido da entrevista semiestruturada, sem deixar de identificar todas as convergências existentes. Posteriormente, a pesquisa foi aprofundada e embasada a partir da literatura pertinente ao assunto (BARDIN, 1997).

\section{RESULTADOS E DISCUSSÃO DOS DADOS}

Após a análise dos depoimentos pelo método proposto, emergiu o tema "Mudanças ocorridas na vida dos ostomizados". Este deu origem a outros subtemas, a saber: "Discriminação"; "Vida sexual"; e "Alimentação", que serão discutidos a posteriori.

\section{DISCRIMINAÇÃO}

Esta categoria mostra, de forma clara e objetiva, a discriminação e vergonha sofridas pelos pacientes. Essa segregação afeta psicologicamente a vida dos indivíduos ostomizados, podendo levar até a uma complicação mais severa. Vejamos abaixo os seguintes discursos:
[...] Mudou muitas coisa, em termo assim que a gente não pode ir numa praia porque é discriminado, a gente não pode chegar em certos ambientes porque a bolsa pode romper aí o mal cheiro fica causano dano, não tanto pra gente como pros outros, aí fica meio difícil, aí acho que seja assim. Até a maioria das pessoas acho que não aceita a gente como se fosse ser humano; acho que tem racismo. É envolvido assim como racismo. Tem gente que vê que você tem uma colostomia aí fica e se afasta de você, fica com nojo de você, fica com ar diferente. É chato, é chato demais a pessoa com uma colostomia. (A)

\section{[...] Nadinha, só assim que eu não} gosto que ninguém saiba que eu tenho porque o povo fica tudo falando. (B)

Nos relatos acima, os pacientes ostomizados descrevem incômodo com o estoma diante da sociedade ou das pessoas que o circundam. A imagem mutilante que eles apresentam no abdômen interfere na vida pessoal e profissional em consequência dos padrões de vida, da busca do corpo perfeito e do alto valor da estética corporal que hoje imperam na sociedade e que levam as pessoas a os discriminarem. 
Além disso, a incapacidade de ter um controle perfeito dos esfíncteres leva o ostomizado a passar por situações desagradáveis no seu meio social.

Desta forma, o individuo que é portador de um corpo imperfeito (ostomia/ostomizado) pode se afastar da sociedade devido às incapacidades $\mathrm{e}$ impossibilidades corporais impostas pela patologia, podendo haver, concomitantemente, desenvolvimento de sentimentos de inadequação e de discriminação pela sociedade.

Os padrões criados pela sociedade levam a uma visão de que o indivíduo que tem alguma fraqueza ou deficiência é um ser contagioso, estragado ou diminuído. Sendo assim, em qualquer situação onde o indivíduo se apresente de forma diferente, pode haver afastamento da relação social devido à característica apresentada distinta daquela prevista pela sociedade (SANTOS, 2005).

Nesta mesma linha de pensamento, as mudanças no modo de vida decorridas após a realização da cirurgia podem gerar reações emocionais como ansiedade, depressão, vergonha e medo de ser rejeitado, fazendo com que haja isolamento social (CESARETTI; LEITE, 2005).

A discriminação hoje sofrida pelos ostomizados é uma importante preocupação a ser analisada e discutida entre os profissionais da saúde, que podem tentar solucioná-la de uma forma multidisciplinar, proporcionando uma melhor qualidade de vida aos mesmos.

\section{VIDA SEXUAL}

Os autores constaram que a confecção das ostomias tiveram implicações sexuais para os pacientes. $\mathrm{O}$ constrangimento social e pessoal com o seu (sua) cônjuge pode ser visulizado no discurso abaixo:

[...] Mudou muita coisa, se não eu já tinha morrido. O ruim é que agora eu não posso mais fazer a única brincadeira que pobre pode fazer, né? A bichinha não se anima mais não; já tomei todo tipo de remédio, mas não tem jeito não, o negócio agora é se conformar. E às vezes a minha esposa não quer chegar perto de mim, não quer nem me limpar; eu é que tenho que fazer isso sozinho. (C)

As alterações na função sexual dos ostomizados estão sendo cada vez mais descritas pelos portadores, pois ocorre perda da libido, diminuição ou ausência da capacidade de ereção e alterações na ejaculação. Esses distúrbios podem ser decorrentes de lesões nervosas do ato operatório (o sistema nervoso autônomo é composto de fibras que têm íntima relação com o 
reto e que têm um importante papel no mecanismo da micção, ereção e ejaculação), como também do conceito da autoimagem comprometido devido à aspectos relacionados a bolsas, odor, secreções e eliminação de gases (SOUSA et al., 1997).

Essas disfunções, ocasionadas pela cirurgia, podem levar a sérias complicações no relacionamento interpessoal com o parceiro, podendo resultar em desintegração familiar. "A privação do sexo é tida como uma das fortes causas da depressão" (OLIVEIRA; NAKANO, 2005).

\section{ALIMENTAÇÃO}

O discurso que será apresentado a seguir mostra as alterações comportamentais referentes à alimentação diária de um portador de estoma. Além disso, aponta o nível de conhecimento do ostomizado quanto ao funcionamento do estoma através da alimentação. Vejamos o relato:

[...] Tem que comer, né? Só não pode é parar de comer, e a minha é pior porque deu aquela entrada e é bem pequenininho o orifício, aí a alimentação eu tenho que balancear; se eu comer um laxante hoje, aí no outro dia eu tenho que comer alguma coisa pra prender mais, mas dá pra viver. (D)
Nota-se no depoimento uma maior preocupação com os alimentos a serem ingeridos, já que alguns deles causam produção de gases excessiva, resultando, assim, em desconforto social. Esse incômodo pode ser minimizado quando ocorre uma intervenção multiprofissional. A partir disso, há uma melhor adaptação à nova nutrição a ser ingerida pelos ostomizados.

A dieta proposta às pessoas portadoras de colostomias advém de uma orientação alimentar e de um esclarecimento acerca dos alimentos a ser ingeridos e suas reações, assim como também dos hábitos alimentares a serem seguidos. Os profissionais de saúde devem esclarecer os ostomizados quanto aos alimentos que causam odor, diarreia, flatulência e obstipação intestinal, para que os mesmos não venham a passar por algum desconforto social (SANTOS, 1997).

\section{CONCLUSÃO}

Diante do exposto, percebe-se que há uma grande dificuldade de reinserção de ostomizados, já que os mesmos apresentam constrangimento, vergonha e afastamento do convívio social após o procedimento cirúrgico.

O consequente uso da bolsa de colostomia leva o indivíduo a desenvolver sentimento de inadequação 
devido à falta de controle dos gases. Além disso, a defecação incontrolável leva o colostomizado a mudar os rumos de sua vida, já que, após essa cirurgia, eles se sentem incapazes de realizar atividades físicas, de adequar um novo balanço nutricional em sua alimentação cotidiana e de vivenciar a sexualidade.

É mister alertar os profissionais de saúde a colocar em prática os cuidados necessários a estes pacientes, incentivando $\mathrm{o}$ retorno às suas atividades rotineiras. Com isso, haverá diminuição do medo, da vergonha e do preconceito que sofrem diante da sociedade.

\section{REFERÊNCIAS}

1. BARDIN, L. Análise de conteúdo. $1^{\circ}$ edição. Lisboa: Edições 70; 1997.

2. CESARETTI, Isabel Umbelina Ribeiro; LEITE, Maria das Graças. Bases para o Cuidar em Estomaterapia. In: SANTOS, Vera Lúcia Conceição de Gouveia; CESARETTI, Isabel Umbelina Ribeiro. Assistência em Estomoterapia.. São Paulo: Atheneu, 2005.
Enfermagem na trajetória do Ostomizado: Pré \& Trans \& PósOperatórios. In: SANTOS, Vera Lúcia Conceição de Gouveia; CESARETTI, Isabel Umbelina Ribeiro. Assistência em estomoterapia. São Paulo: Atheneu, 2005.

4. DIAS, Maria Carolina Gonaçalves; TEIXEIRA, Cláudia de Fátima Gomes. Intervenção Nutricional no ostomizado. In: SANTOS, Vera Lúcia Conceição de Gouveia; CESARETTI, Isabel Umbelina Ribeiro. Assistência em Estomoterapia. São Paulo: Atheneu, 2005.

5. DUGAS, Beverly Witter. Enfermagem Prática. Rio de janeiro: Guanabara. $4^{\circ}$ edição; 1998.

6. GEMELLI, Lorena Morais Goetem; ZAGO, Marcia Maria Fontão. A interpretação do cuidado com o ostomizado na visão do enfermeiro: um estudo de caso. Revista LatinoAmericana de Enfermagem. V. 10, $\mathrm{n}^{\circ}$ 1, Ribeirão Preto, jan 2002. p. 35. Disponível em: http://www.scielo.br/pdf/rlae/v10n1/7 769.pdf. Acesso em: 22 de abr.2009.

3. CESARETTI, Isabel Umbelina Ribeiro et al. O Cuidar de 
7. GIL, Antonio Carlos. Como Elaborar Projetos de Pesquisa. $4^{\circ}$ edição, São Paulo. Atlas, 2002.

8. MENDONÇA, Regiane de Souza et al. A Importância da Consulta de Enfermagem em Pré-operatório de Ostomias Intestinais. Revista Brasileira de Cancerologia. Rio de Janeiro, 2007. p. 434. Disponível em: http://www.inca.gov.br/rbc/n_53/v04/ pdf/artigo5.pdf. Acesso em : 28 mar.2009.

9. MINAYO, Maria Cecilia de Souza; Pesquisa qualitativa em saúde Desafio do Conhecimento. 17 ed. Rio de Janeiro, ed. Vozes, 2006.

10. NETTINA, Sandra M.. Prática de Enfermagem. Rio de Janeiro: Guanabara, 7. ed. 2003. p. 575.

11. OLIVEIRA, Dirce Venancio Domingues; NAKANO, Tereza Tamiko Yara. Reinserção Social do Ostomizado. In: SANTOS, Vera Lúcia Conceição de Gouveia; CESARETTI, Isabel Umbelina Ribeiro. Assistência em Estomoterapia.. São Paulo: Atheneu, 2005.
12.POTTER, Patricia A.; PERRY, Anne Griffin. Fundamentos de Enfermagem. São Paulo: Artmed, $5^{\circ}$ edição, 2004.

13. SANTOS, Solange Pereira. Nutrição em portadores de estoma. In: Org: CREMA, Eduardo; SILVA, Rosemary ESTOMAS Uma abordagem Interdisciplinar.. $1^{\circ}$ edição. Minas Gerais: Pinti, 1997.

14. SANTOS, Vera Lúcia Conceição de Goveia. A estomoterapia Através dos Tempos. In: SANTOS, Vera Lúcia Conceição de Gouveia; CESARETTI, Isabel Umbelina Ribeiro. Assistência em estomoterapia.. São Paulo: Atheneu, 2005.

15.SANTOS, Vera Lúcia Conceição de Goveia. Representações do Corpo e a Ostomia. Estigma. In: : SANTOS, Vera Lúcia Conceição de Gouveia; CESARETTI, Isabel Umbelina Ribeiro. Assistência em Estomoterapia. São Paulo: Atheneu, 2005.

16. SCHIMIDT, T. C. G; ARRUDA, M. L. Sentimentos da família na interação com a equipe de enfermagem. Cogitare Enferm. 2012; 17(2):348-54. Disponível em: ojs.c3sl.ufpr.br/ojs/index.php/cogitare/ 
article/download/27898/18554.

Acesso em: 14 de maio de 2014.

17. SOUSA, João Batista de et al. Implicações Sexuais na cirurgia do Estoma Intestinal. In: CREMA, Eduardo; SILVA, Rosemary. ESTOMAS Uma abordagem Interdisciplinar.. $1^{\circ}$ edição. Minas Gerais: Pinti, 1997.

18.SWARINGEN, Pamela L.; HOWARD, Cheri A. Atlas Fotográfico de Procedimentos de Enfermagem. Rio de Janeiro:

Guanabara, $3^{\circ}$ edição, 2004. 